\title{
Optimum Strategy for Siting of New Tidal Stations in the Bohai Sea
}

\author{
Song Gao, Jiangling $\mathrm{Xu}^{*}$, Lingjuan $\mathrm{Wu}$ and Dongling Guo \\ North China Sea Marine Forecasting Center of State Oceanic Administration, Qingdao 266033, China \\ Shandong Provincial Key Laboratory of Marine Ecological Environment and Disaster Prevention and Mitigation, Qingdao \\ 266033, China \\ *Corresponding author
}

\begin{abstract}
A two-dimensional tidal model with the adjoint method is used to investigate the optimum strategy for siting of new tidal stations in the Bohai Sea. Open boundary conditions of the model are estimated and the M2 constituent in the Bohai Sea is simulated by assimilating observations from tidal stations and T/P altimeter. Harmonic constants (HCs) at grids on the coastline computed by assimilating real observations serve as observed values for subsequent experiments, in which HCs at grids on the coastline are estimated by assimilating observed values of all tidal stations with different strategies. By analyzing the mean vector difference (MVD) between HCs' observed and inverted values on the coastline, the optimum strategy for siting of new tidal stations was obtained. To investigate the influence of uniform and nonuniform siting, experiments with different principle are carried out. Results indicate that the uniform siting is more effective than nonuniform siting.
\end{abstract}

Keywords-open boundary conditions; adjoint method; optimization strategy; tidal stations; two-dimensional

\section{INTRODUCTION}

A major difficulty in tidal simulation is determination of open boundary conditions (OBCs). Traditionally, OBCs can be obtained from global tidal estimates, larger scale models, or extrapolation from available observations. However, experience is required when the methods mentioned above are used. The adjoint method based on the theory of inverse problems is a powerful tool for parameter estimation with the advantage of assimilating various observations distributed in time and space. OBCs of a tidal model can be optimized automatically by assimilating observations from satellite altimetry and tidal stations using the adjoint method. Lardner $[1,2]$ noted OBCs maybe obtained by assimilating observations in a 2-D depth-averaged model. Seiler [3] did a related study with a quasi-geostrophic, open-ocean model. Lvet al. [4,5] derived $\mathrm{M}_{2}$ tidal harmonic constants on the OBCs of the Bohai, Yellow and East China Seas using adjoint method and the harmonic constants at tidal stations in the interior of the region. He et al. [6] investigated shallow water tidal constituents in the Bohai and Yellow Seas by assimilating T/P altimeter data with the adjoint method. Zhang and Lv[7] simulated threedimensional tidal current sin the marginal seas by assimilating satellite altimetry data. They designed twin experiments to demonstrate reasonability and feasibility of the model, and estimated OBCs in practical experiments.
In this paper, $\mathrm{OBCs}$ and $\mathrm{HC}$ of $\mathrm{M}_{2}$ constituent at all computing grids in the Bohai Sea are estimated by assimilating tidal station data and $\mathrm{T} / \mathrm{P}$ altimeter data where water depth is more than $30 \mathrm{~m}$. To improve the results of numerical simulation, the prescribed bottom friction coefficients were also adjusted by adjoint assimilation. HCs grids on the coastline computed by assimilating real observations serve as observed values for subsequent experiments, in which HCs at grids on the coastline are estimated by assimilating observed values of all tidal stations with different strategies. This paper is organized as follows: The tidal model is introduced in Section 2. In Section 3, two groups of numerical experiments are performed, investigating influence of step-length on the inversion results, MVD of different strategies in practical experiment on the inversion results. Conclusions are given in Section 4.

\section{TIDAL MODEL AND SETTINGS}

Assuming pressure is hydrostatic and density is constant, the 2-D tidal model used is as follows:

$$
\begin{aligned}
& \frac{\partial \zeta}{\partial t}+\frac{\partial[(h+\zeta) u]}{\partial x}+\frac{\partial[(h+\zeta) v]}{\partial y}=0 \\
& \frac{\partial u}{\partial t}+u \frac{\partial u}{\partial x}+v \frac{\partial u}{\partial y}-f v+\frac{k u \sqrt{u^{2}+v^{2}}}{h+\zeta}-A\left(\frac{\partial^{2} u}{\partial x^{2}}+\frac{\partial^{2} u}{\partial y^{2}}\right)+g \frac{\partial \zeta}{\partial x}=0 \\
& \frac{\partial v}{\partial t}+u \frac{\partial v}{\partial x}+v \frac{\partial v}{\partial y}+f u+\frac{k v \sqrt{u^{2}+v^{2}}}{h+\zeta}-A\left(\frac{\partial^{2} v}{\partial x^{2}}+\frac{\partial^{2} v}{\partial y^{2}}\right)+g \frac{\partial \zeta}{\partial y}=0
\end{aligned}
$$

where $t$ is time, $x$ and $y$ are Cartesian coordinates (positive eastward and northward, respectively), $h(x, y)$ is undisturbed water depth at location $(x, y), \zeta(x, y)$ is sea surface elevation above the undisturbed sea level, $u(x, y, t)$ and $v(x, y, t)$ are velocity components in the horizontal $x$ - and $y$-directions, $f$ is the Coriolis parameter, $k$ is the bottom friction coefficient, and $A$ is the coefficient of horizontal eddy viscosity.

Initial conditions are such that the initial sea surface elevation, $x$ - and $y$-velocities are zero. The closed boundary conditions are such that the normal velocity is zero, and OBCs are sea surface elevations on the open boundary, which is as follows:

$$
\zeta=a \cos (\omega t)+b \sin (\omega t)
$$

where $a$ and $b$ are the Fourier coefficients on the open 
boundary, and $\omega$ is the angular frequency of the $\mathrm{M}_{2}$ constituent.

The cost function is constructed as

$$
J=\frac{1}{2} K_{\zeta} \sum_{j=1}^{i t e} \sum_{(m, n) \in D_{\zeta}}\left(\zeta_{m, n}^{j}-\hat{\zeta}_{m, n}^{j}\right)^{2}
$$

where $K_{\zeta}$ is a constant (here, $K_{\zeta}=1$ ), $D_{\zeta}$ is the set of observation locations, $\zeta_{m, n}^{j}$ is the simulated result, $\hat{\zeta}_{m, n}^{j}$ is the observation and ite is the number of time steps of the forward model.

The finite difference scheme used in this study is similar to Lv and Zhang [8], except that we use the rectangular coordinate instead of spherical coordinate.

The region of interest is the Bohai Sea and the open boundary is at $121.25^{\circ} \mathrm{E}$. Horizontal resolution of the model is $5^{\prime} \times 5^{\prime}$ and the time step is 93.154 seconds, which is $1 / 480$ of the period of the $\mathrm{M}_{2}$ constituent. The tidal gauge data, T/P altimeter data, bottom friction coefficient and horizontal eddy viscosity coefficient are the same as in Lv and Zhang [8]. A bathymetry map of the Bohai Sea and observation sites are shown in Figure 1.

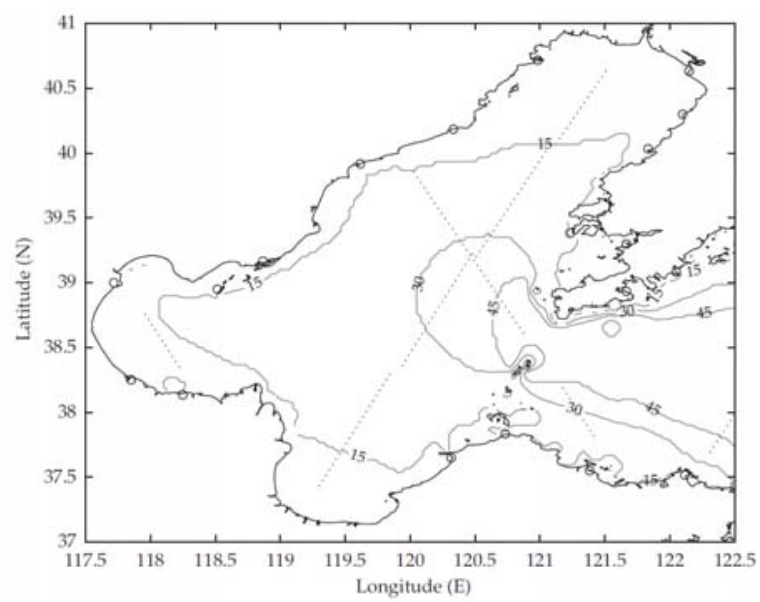

FIGURE I. BATHYMETRY MAP OF BOHAI SEA AND LOCATION OF OBSERVATIONS (T/P ALTIMETER TRACKS ARE DENOTED BY DOTS AND TIDAL GAUGES BY CIRCLES).

\section{NUMERICAL EXPERIMENTS AND RESULTS ANALYSIS}

A method is put forward to determine the optimum strategy for siting of new gauge stations, which is described as follows:

1)To ensure that new gauge stations and the old ones are distributed as evenly as possible on the coastline of the Bohai Sea, set a series of strategies $\left(E X_{i}\right)$ in different provinces' coastline, where $i$ represents the serial number of strategies.

2) Two-dimensional tidal open boundary conditions (Figure 2), the siting of bottom friction coefficient (Figure 3)and $\mathrm{HC}$ of M2 constituent at all computing grids (Figure 4) are estimated with tidal station data and $\mathrm{T} / \mathrm{P}$ altimeter data where water depth is more than $30 \mathrm{~m}$. HCs at coastline grids serve as observed values $(H 0, j, \theta 0, j)$ in the following groups of experiments, $\mathrm{j}$ represents the serial number of grid at coastline.

3) HCs ( $H i j, \theta i, j)$ are computed by assimilating the observed values, where $\mathrm{i}$ represents the serial number of different strategy, $j$ represents the serial number of grid at coastline.

4) The mean vector difference (MVD) between $\left(H_{0, j}, \theta_{0, j}\right)$ and $\left(H_{i, j}, \theta_{i, j}\right)$ is calculated as:

$$
\Delta r_{i}=\frac{1}{N} \sum_{j=1}^{N} \sqrt{H_{i, j}{ }^{2}+H_{0, j}{ }^{2}-2 H_{0, j} H_{i, j} \cos \left(\theta_{i, j}-\theta_{0, j}\right)},
$$

where $N$ represents the total number of coastline grids in the each compared area (Fig.5), $\left(H_{0, j}, \theta_{0, j}\right)$ and $\left(H_{i, j}, \theta_{i, j}\right)$ is also in the compared area. Because the MVD reflects the field-average deviation of model results, when the MVD is sufficiently small (smaller than the other strategies), and the corresponding strategy is regarded as the optimum.

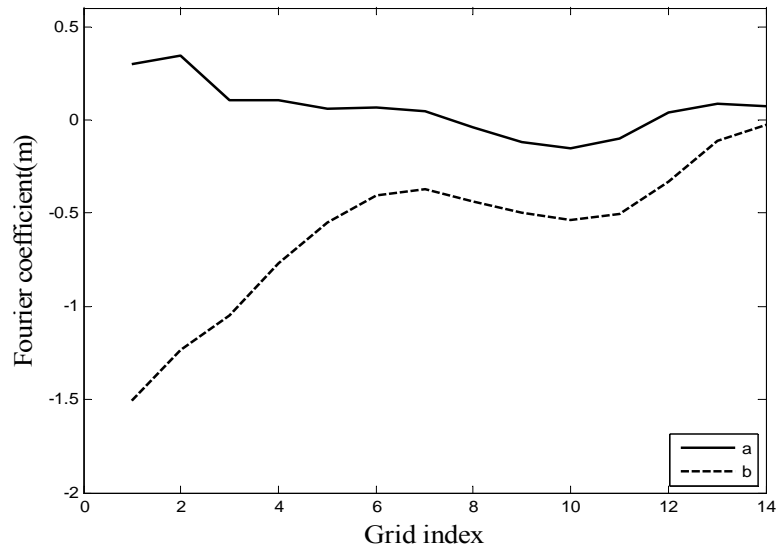

FIGURE II. FOURIER COEFFICIENTS $(a, b)$ OF THE M2 CONSTITUENT AT $121.25^{\circ} \mathrm{E}$

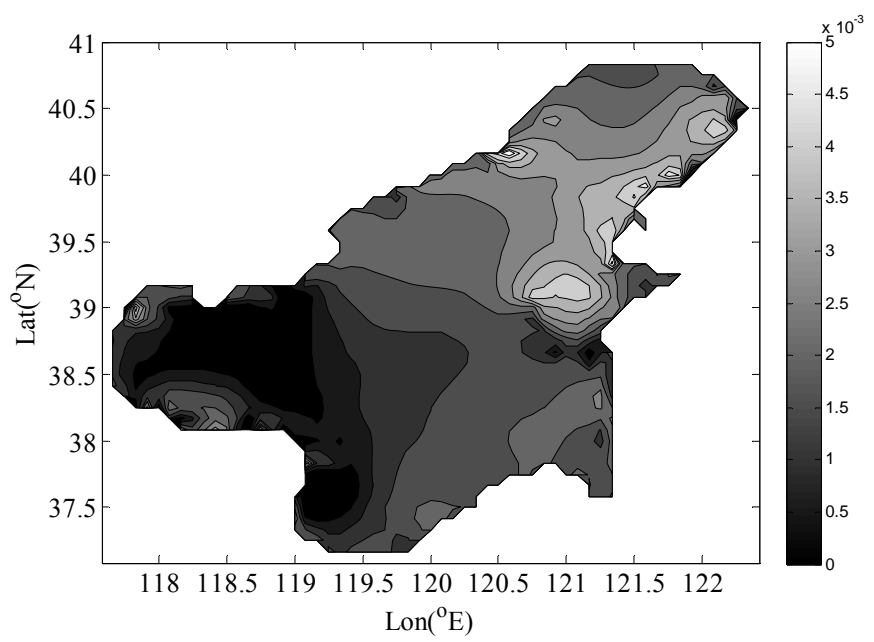

FIGURE III. THE DISTRIBUTION OF BOTTOM FRICTION COEFFICIENT IN THE BOHAI SEA 


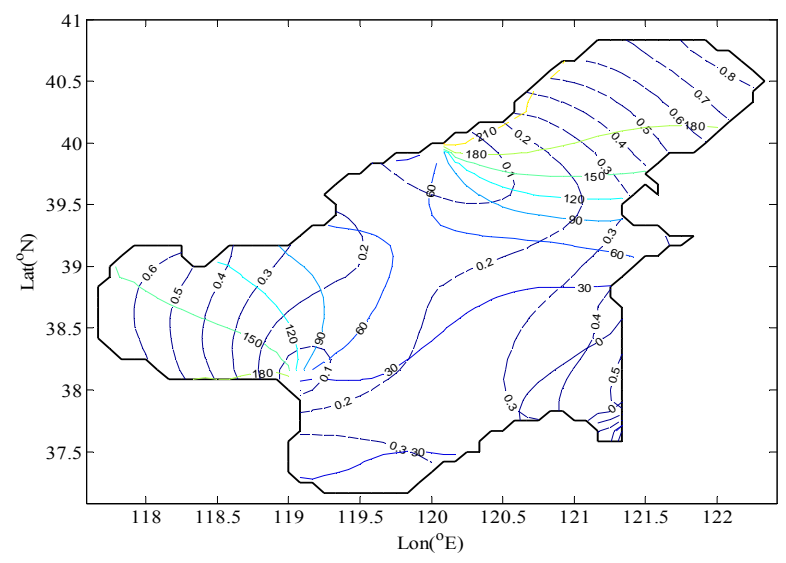

FIGURE IV. CO-TIDAL CHARTS OBTAINED WITH TIDAL STATION DATA AND T/P ALTIMETER DATA (DASHED LINE DENOTES COAMPLITUDE LINE (M), AND SOLID LINE DENOTES THE CO-PHASE $\left.\operatorname{LINE}\left({ }^{\circ}\right)\right)$

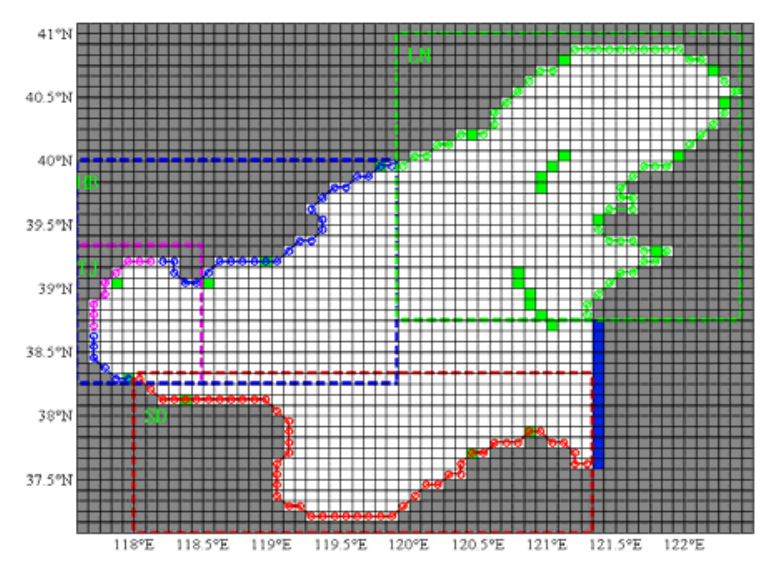

FIGURE V. GRID MAP OF DIFFERENT COMPARED AREA (THE BLUE GRIDS REPRESENT OPEN BOUNDARY, THE GREEN GRIDS REPRESENT TIDAL STATIONS AND POINTS ON THE T/P SATELLITE TRACKS WHERE WATER DEPTH IS MORE THAN 30M, THE GRAY GRIDS REPRESENT DRY GRID, AND THE WHITE REPRESENTS WET GRIDS)

\section{A. Group 1}

The purpose of Group1 is to investigate the influence of step-length on inversion results. In parameter optimization, we tried 100 iterations. We choose the gradient decedent algorithm to estimate the Fourier coefficient $a$ and $b$ in the following groups of experiments [9]. The step-length is set to 0.25 as a matter of priority [10].The control parameter sin the 2-D tidal model can be optimized using the following formulas:

$$
X_{\text {new }}=X_{\text {old }}-\alpha \cdot\left(\frac{\partial J}{\partial A}\right)^{0},
$$

Where $J$ is the cost function which quantifies the discrepancy between the model results and the observed values, and $\left(\frac{\partial J}{\partial A}\right)^{0}\left(\left(\frac{\partial J}{\partial A}\right)^{0}=\frac{\partial J / \partial A}{\|\partial J / \partial A\|}\right)$ denotes the normalized gradient with respect to $A$. $\alpha$ is step-length which is used to adjust the parameters preferably. $\alpha$ is determined with the following six strategies, in which only existing tidal stations are used. When mean absolute errors (MAEs) of amplitude and phase between simulated and observed values are less than $5 \mathrm{~cm}$ and $5^{\circ}$, respectively, we conclude that the two-dimensional tidal model with adjoint method estimates $\mathrm{HC}$ successfully.

Strategy $1: \alpha=0.25$;

Strategy2: $\alpha=0.25 \times 0.99^{m}$;

Strategy3: $\alpha=0.25 \times 0.98^{m}$;

Strategy4: $\alpha=0.25 \times 0.97^{\mathrm{m}}$;

Strategy5: $\alpha=0.25 \times 0.96^{\mathrm{m}}$;

Strategy $6: \alpha=0.25 \times 0.95^{m}$;

where $m$ denotes the $m$ th iteration.

Table 1 shows differences between HCs' simulated and observed values in Group $1, \Delta H$ and $\Delta g$ represent MAEs of amplitude and phase, and $\Delta r$ represent MVD, respectively. The variation curves of cost functions and their norms are shown in Figure 6 . The cost functions obtained with all six strategies decline remarkably by 5 orders of magnitude after

\section{TABLE I. DIFFERENCES BETWEEN SIMULATED AND OBSERVED VALUES}

\begin{tabular}{crrrrrr}
\hline Strategies & $\mathbf{1}$ & $\mathbf{2}$ & $\mathbf{3}$ & $\mathbf{4}$ & $\mathbf{5}$ & $\mathbf{6}$ \\
\hline$\Delta H(\mathrm{~cm})$ & 3.48 & 1.06 & 1.04 & 1.15 & 1.09 & 1.19 \\
$\Delta g\left({ }^{\circ}\right)$ & 2.36 & 1.60 & 1.08 & 1.10 & 1.09 & 1.46 \\
$\Delta r(\mathrm{~cm})$ & 4.51 & 2.94 & 1.55 & 1.61 & 1.57 & 1.90 \\
\hline
\end{tabular}

100 iterations. The step-length has an effect on inversion results. The gradients of the cost functions with respect to Fourier coefficient $(a, b)$ decline remarkably with six strategies. The MVD obtained with strategy 3 is $1.55 \mathrm{~cm}$, which is the minimal among those for all strategies. The MVD obtained with strategy 5 is $1.57 \mathrm{~cm}$, which is very close to that with strategy 3 . The variation curve of cost function with strategy 5 is more smooth than strategy 3, indicating that strategy 5 is more effective. According to the results, the best assimilation efficiency can be achieved with strategy 5 , i.e., the step-length is $0.25 \times 0.96^{m}$. Therefore we choose $\alpha=0.25 \times 0.96^{m}$ to estimate the Fourier coefficient $(\mathrm{a}, \mathrm{b})$ in the following groups of experiments.
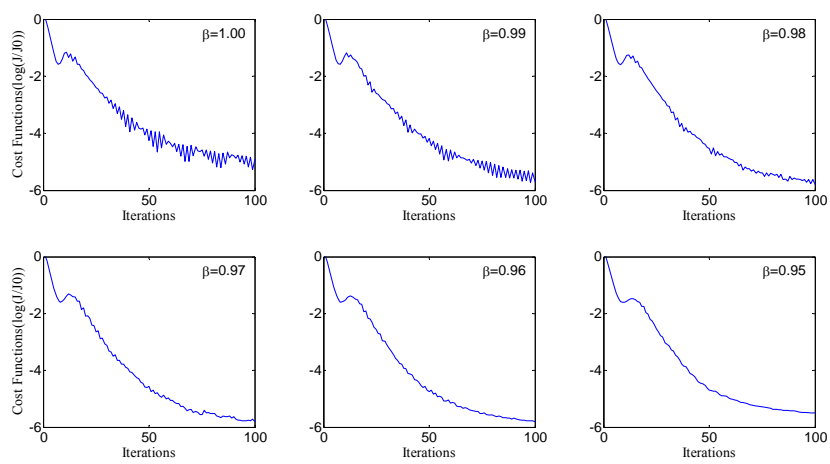

FIGURE VI. VARIATION CURVES OF COST FUNCTIONS IN GROUP 1

\section{B. Group 2}

In this section, the optimal strategy for the coastline in 
Liaoning Province is selected from twelve strategies. There are 62 computing grids, 7 existing tidal stations, and 7 tidal stations to be sited on the coastline in Liaoning Province in the Bohai Sea. A tidal station is sited every 4 or 5 computing grids for uniform siting. Therefore, twelve strategies (1 12) are designed. Strategy 13, in which only observed values from existing stations are assimilated, is carried out to investigate influence of increasing new tidal stations (Figure 7).

As shown in Table 2, the MVD of all strategies with new tidal stations is smaller than strategy 13, indicating that assimilation is improved by increasing tidal stations. Station location has an effect on inversion results. the MVD of $\mathrm{M}_{2}$ constituent obtained with strategy 2 is $2.04 \mathrm{~cm}$, which is the minimal among those for all strategies. This indicates that strategy 2 is more effective than the others. Therefore strategy 2 will be employed in the next experiment.

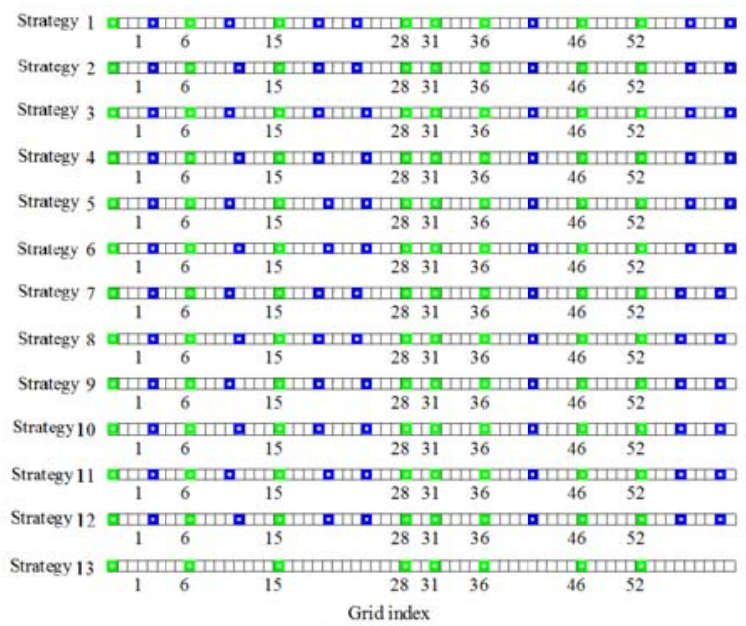

FIGURE VII. THIRTEEN DIFFERENT STRATEGIES FOR LIAONING PROVINCE COAST (THE GREEN GRIDS REPRESENT EXISTING TIDAL STATION AND THE BLUE GRIDS REPRESENT NEW TIDAL STATION)

TABLE II. DIFFERENCES BETWEEN SIMULATED AND OBSERVED VALUES IN GROUP 2

\begin{tabular}{cccc}
\hline Strategies & $\boldsymbol{\Delta} \boldsymbol{H}(\mathbf{c m})$ & $\boldsymbol{\Delta}\left({ }^{\circ}\right)$ & $\Delta \boldsymbol{r}_{\boldsymbol{i}}(\mathbf{c m})$ \\
\hline 1 & 0.80 & 1.14 & 2.05 \\
2 & 0.80 & 1.13 & 2.04 \\
3 & 0.82 & 1.17 & 2.11 \\
4 & 0.81 & 1.15 & 2.07 \\
5 & 0.81 & 1.16 & 2.08 \\
6 & 0.80 & 1.15 & 2.06 \\
7 & 1.51 & 1.30 & 2.71 \\
8 & 1.51 & 1.29 & 2.70 \\
9 & 1.52 & 1.34 & 2.78 \\
10 & 1.52 & 1.33 & 2.76 \\
11 & 1.52 & 1.34 & 2.78 \\
12 & 1.51 & 1.33 & 2.76 \\
13 & 2.06 & 1.22 & 2.88 \\
\hline
\end{tabular}

\section{CONCLUSION}

According to the results of the four groups of experiments, the optimum strategy for siting of new tidal stations on the coastline in the Bohai Sea can be obtained by assimilating tidal station data and T/P altimeter data into a two-dimensional tidal model with the adjoint method.

In Group 1,six strategies of step-length are tested, with which the inversion results of $\mathrm{HC}$ are satisfactory. It proves that the step-length of $0.25 \times 0.96^{\mathrm{m}}$ improves the assimilation efficiency. In Group 2, different strategies of tidal siting in Liaoning Province is investigated. The results indicate that the minimal MVD can be obtained with strategy 2, which can be determined as the optimum strategy for Liaoning Province.

Based on group 2, optimum strategy for siting of new tidal stations on the coastline in Tianjin Municipality, Shandong and Hebei Province can be developed, respectively. Combination of optimum strategies for the four provinces serves as the optimum one for the Bohai Sea(Fig. 8).

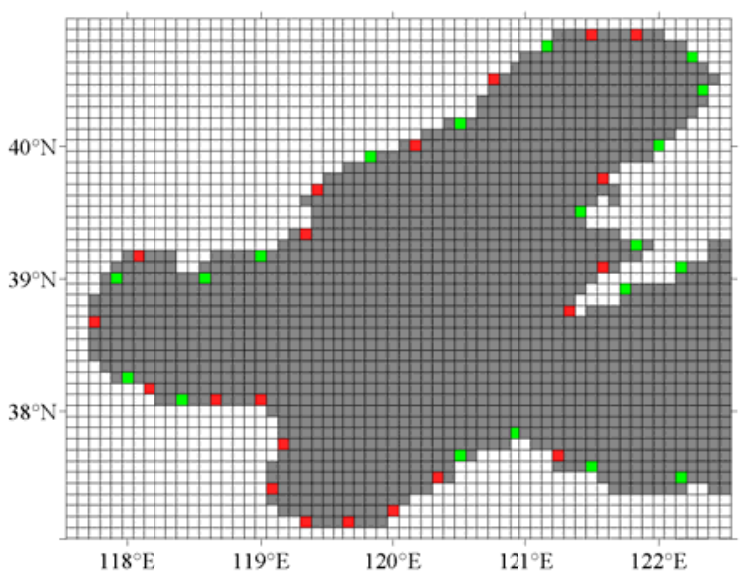

FIGURE VIII. GRID MAP OF NEW TIDAL STATION IN THE BOHAI SEA (RED GRIDS REPRESENT NEW TIDAL STATIONS AND THE GREEN GRIDS REPRESENT EXISTING TIDAL STATIONS)

\section{ACKNOWLEDGEMENTS}

Partial support for this research was provided by the Marine Public Foundation through grant 2013418026 and 201305003, the National High Technology Research and Development Program of China(863 Program) through grant 2013AA122803, the National Science Foundation of Shandong Province, China through grant ZR2014DM017, and the Natural Science Foundation of Zhejiang Province through Grant LY15D060001.

\section{REFERENCES}

[1] Lardner R. W., Optimal control of an open boundary conditions for a numerical tidal model. Computer Methods in Appl. M., 1993, 102: $367 \sim 387$.

[2] Lardner R. W., Optimal assimilation of current and surface elevation data in a two-dimensional numerical tidal model. Appl. Math. Modelling. 1995, 19: 30 38.

[3] Seiler U., Estimation of open boundary conditions with the adjoint method. J. Geophys .Res., 1993, 98: 22855 22870.

[4] Lv X. Q. and Fang G. H., Numerical experiments of the adjoint model for M2tide in the Bohai Sea .Acta. Oceanologica Sincia. 2002, 24 : 7 245

[5] Lv X. Q., Wu Z. K., Yin Z. B. and Tian J. W., Inversion of the Tides with the Open Boundaries of the Bohai, Huanghai and Donghai Seas. Journal of Ocean University of Qingdao, 2003, 33: 164 172

[6] He Y. J., Lv X. Q., Qiu Z. F., Zhao J P. Shallow water tidal constituents in the Bohai and the Yellow Sea from an umerical adjoint model with 
TOPEX/POSEIDON altimeter data. Cont .Shelf.Res .2004. , 24 : 1 5211529.

[7] Zhang J. C., Lv X. Q., Inversion of three-dimensional tidal currents in marginal seas by assimilating satelliteal timetry. Comput. Method. Appl. M.2010., 199: 3 125-3 136.

[8] Lv X. Q., Zhang J. C., Numerical study on spatially varying bottom friction coefficient of a 2D tidal model with adjoint method. Cont . Shelf. Res .2006, 26: 1905-1923.

[9] Zhang Q. L. Gao Y. Q. and Lv X. Q., Estimation of Oceanic Eddy Viscosity Profile and Wind Stress Drag Coefficient Using Adjoint Method. Math. Probl. Eng. 2015(2015). Article ID 309525

[10] Guo Z., Cao A. Z. and Lv X. Q., Inverse Estimation of Open Boundary Conditions in the Bohai Sea. Math. Probl .Eng. 2012(2012). Article ID 628061 . 